\title{
La partitura como testimonio de la tradición. Un caso sobre identidad y etnomusicología en la historia
}

\author{
The Score as a Testimony of Tradition. A Case about \\ Identity and Ethnomusicology in History
}

En el presente trabajo se propone el empleo de obras de música académica como fuente para el estudio de géneros populares andinos en la época colonial tardía y albores de la república en los actuales territorios de Perú y Bolivia, época difícil de documentar debido a la escasez de fuentes escritas. Este planteamiento podría contribuir al estudio de fases tempranas de dichos géneros, cómo difieren de sus versiones contemporáneas, documentar aquellos que ya han desaparecido y la forma en que autores académicos plasmaron en sus obras el material de origen tradicional. Se emplea como ejemplo de estudio el tercer y quinto movimiento del Divertimento Concertante Opus 43 para guitarra, dos flautas y cuarteto de cuerdas del compositor peruano-boliviano Pedro Ximénez Abrill y Tirado (Arequipa, Perú, 1784?; Sucre, Bolivia 1856)

Palabras clave: Pedro Ximénez Abrill, yaraví, vals, música popular del Siglo XIX, identidad.

This article proposes the use of works of art music as a source for the study of Andean popular genres in the late colonial period and the dawn of the republic in the present-day Peru and Bolivia, a difficult period to document due to the scarcity of written sources. This approach could contribute to the study of the early phases of such genres, how they differ from their contemporary versions, to documenting those that have already disappeared, and how academic composers have reflected material of a traditional origin into their works. The third and fifth movements of the Divertimento concertante, op. 43 for guitar, two flutes and string quartet by the Peruvian-Bolivian composer Pedro Ximénez Abrill y Tirado (Arequipa, Peru, 1784?; Sucre, Bolivia, 1856) are used as a case study.

Keywords: Pedro Ximénez Abrill, harawi, waltz, popular music of the 19th century, identity.

\section{Introducción}

En 2005, María Madrazo realizó algunas reflexiones sobre el término "tradición" y sus definiciones desde diversas disciplinas y conceptos relacionados al cambio, transmisión e incluso olvido de los contenidos culturales

\footnotetext{
${ }^{1}$ El presente trabajo es parte de la investigación doctoral titulada La composición académica como fuente testimonial del género popular: el yaraví temprano en la obra de Pedro Ximénez de Abrill y Tirado que la autora realiza como alumna del programa de doctorado en musicología de la Facultad de Música de la Universidad Nacional Autónoma de México (UNAM).
} 
considerados tradicionales al interior de las comunidades. Ella definía la tradición como

un proceso de transmisión, que viene del pasado al presente, se realiza mediante una cadena de repeticiones que no son idénticas, sino que presentan cambios e innovaciones, y se van acumulando para crear lo que sería la gran tradición, un acervo reunido a lo largo de las repeticiones y que abarca las diferentes versiones de la transmisión ${ }^{2}$.

Madrazo explica que, desde el punto de vista sociológico y antropológico, la tradición tiene un significado colectivo, que posee conocimientos, prácticas, creencias y valores originados en el pasado, pero esenciales en el presente para establecer la continuidad y cohesión de la comunidad $^{3}$. Por este enraizamiento en el pasado, la tradición alcanza validez y autoridad y, de esta manera, al ser custodiado y conservado por sus poseedores, su contenido crea el presente desde el pasado y se convierte en un signo de identidad en la comunidad a la que pertenece. No obstante, los miembros de este mismo grupo pueden moldearla, adaptarla y transformarla según sus necesidades, por lo que la tradición está, en realidad, en cambio permanente ${ }^{4}$.

Este pasado que proporciona a la tradición su fundamento y autoridad es, en palabras de Caroline Bithell, "una fuente de símbolos culturales que tienen poder más allá de la mera historia" ${ }^{\circ}$ y, por esta razón, el poder de estos símbolos culturales, dentro de los cuales consideramos a los géneros musicales, tendría vigencia no solo en la dimensión histórica, sino en el presente, como indica Madrazo. Así pues, los habitantes buscan emplearlos con mayor asiduidad, según sus necesidades, especialmente porque estas tradiciones del pasado "ofrecen un refugio para las complejidades y confusiones de la vida moderna, al servir como un ancla en la tormenta que amenaza destruir identidades desde sus raíces [...]. Las elecciones musicales pueden ser parte del proceso en el cual la gente en el presente escoge o construye una historia que sustenta sus necesidades actuales" y, además, "al mantener aspectos vivos del pasado, la música también puede funcionar como un símbolo de resiliencia y sobrevivencia [...], el estilo musical define no solo quién es la gente, sino quienes no son" ${ }^{6}$. Una tradición que proporciona herramientas para defender o afianzar la identidad necesariamente

\footnotetext{
2 María Madrazo Miranda: "Algunas consideraciones en torno al significado de la tradición", Contribuciones desde Coatepec, n. ${ }^{\circ}$ 9, 2005, p. 115-132; 123

${ }^{3}$ M. Madrazo: "Algunas consideraciones...", p. 122.

${ }^{4}$ Ibid., pp. 117-127.

${ }^{5}$ Caroline Bithell: "The Past in Music: Introduction", Ethnomusicology Forum, 15, 1, 2006, pp. 3-16; 5 .

${ }^{6}$ Ibid., pp. 5 y 8.
} 
extrae del pasado dichas herramientas para emplearlas en el presente e, incluso, pueden usarse de maneras muy diferentes a sus propósitos originales, transformando contenidos y formas. La necesidad de conservar, transformar u olvidar determinados productos sonoros, podría explicarse por lo que Rice llamó los nuevos significados asignados a viejas formas musicales para satisfacer nuevas demandas ${ }^{7}$. Este trabajo pretende ofrecer algunos ejemplos de cómo estos símbolos culturales, expresados como géneros musicales hoy considerados tradicionales pueden ser historiados desde fuentes escritas tempranas para explicar mejor la asignación de sentido que se les ha dado tanto en el pasado como en el presente.

En la región sur del Perú, en la ciudad de Arequipa, capital de la provincia y región del mismo nombre, perviven varios ejemplos de géneros tradicionales como el yaraví, el vals y la marinera. Sin embargo, los cambios sociales y culturales ocurridos en la ciudad en las últimas cuatro décadas han desafiado los conceptos de identidad y de "lo arequipeño" y los géneros musicales se han visto contrastados por expresiones culturales provenientes de regiones aledañas (zonas alto andinas de Arequipa, Cusco y fundamentalmente Puno), o por las que difunden los medios masivos de comunicación (radio, cine, televisión e internet). Tales expresiones y géneros, desconocidos en la región hasta hace relativamente poco tiempo, buscan espacios y oportunidades en la zona urbana, sobre todo en los distritos de reciente fundación, en contraposición a los pueblos tradicionales y el centro histórico de la ciudad.

En respuesta a este fenómeno, en años recientes se ha suscitado un notorio interés por recuperar, conservar y descubrir raíces culturales denominadas "propias", "tradicionales" o "auténticas" que sustenten la identidad del arequipeño frente a estas influencias foráneas. Los estudios referidos a música, literatura, historia, cultura popular, gastronomía, lingüística y arqueología se han multiplicado en los últimos lustros. Aunque se han hecho aportes notables, la tendencia parece reforzar el sentido de lo que se es (o lo que se piensa que se es) antes de descubrir información nueva o desarrollar nuevas perspectivas de investigación y reflexión sobre la tradición y sus contenidos. Peter Burke ha explicado este fenómeno al afirmar que, en el presente, "se constata un gran interés popular por las memorias históricas. Este interés creciente constituye probablemente una reacción a la aceleración del cambio social y cultural, que amenaza las identidades escindiendo lo que somos de lo que éramos" 8 .

7 Timothy Rice: Ethnomusicology. A Very Short Introduction, Oxford, Oxford University Press, 2014, p. 93

${ }^{8}$ Peter Burke: ¿Qué es historia cultural?, Barcelona, Paidós, 2004, p. 87. 
Los géneros tradicionales a los que recurre la población para afianzar su propia identidad, no conocieron la escritura hasta épocas relativamente recientes. No obstante, algunas referencias pueden encontrarse en obras escritas como lo son el repertorio producido en el ámbito surperuano en la primera mitad del siglo XIX. En este artículo, analizamos el Tercer y Quinto Movimiento del Divertimento Concertante op. 43 para guitarra, dos flautas, dos violines, viola y violonchelo del compositor Pedro Ximénez Abrill y Tirado (Arequipa, Perú 1784?; Sucre, Bolivia 1856), uno de los primeros compositores sudamericanos que emplea formas hoy llamadas tradicionales en música de cámara. En esta obra, Ximénez usa elementos de tres géneros: uno cantado conocido como yaraví y otros dos danzados, el vals y otro al que llamaremos "gallinacito" a falta de otra denominación. En su tiempo, el lenguaje neoclásico coexistía con otras expresiones como formas cantadas de poesía popular y géneros bailables, pero en el presente, el público ha cambiado su manera de decodificar el contenido sonoro de dichas obras al desaparecer las formas bailables como el gallinacito y las convenciones sonoras del clasicismo, y transformarse la forma de escuchar y danzar el vals, mientras que el yaraví se ha convertido en un símbolo de "lo arequipeño" -aunque cada vez es menos cultivado, pero que por eso mismo obtiene mayor valor identitario como defensa contra el cambio cultural, social y económico-. La obra de Pedro Ximénez funciona como un valioso referente para el estudio de dichas formas tradicionales. Con este trabajo nos proponemos reflexionar sobre el aporte que la música escrita puede significar para el estudio de músicas asociadas a la tradición oral.

\section{El Divertimento concertante, op. 43 para Guitarra, dos flautas y cuarteto de cuerdas de Pedro Ximénez Abrill y Tirado}

El compositor y la obra

Pedro Ximénez Abrill y Tirado nació en Arequipa, entonces Virreinato del Perú, muy probablemente en 1784 . Se sabe muy poco de su vida, excepto que era pariente de algunas de las familias más notorias de la ciudad y que, con el tiempo, fue miembro de las instituciones más representativas de la temprana vida republicana como la Academia Lauretana y el Colegio Nacional de la Independencia Americana. Tampoco se sabe quiénes fueron sus maestros, pero se especula que realizó algunos viajes a Lima donde mantuvo contactos con compositores de la época como Andrés Bolognesi y José Bernardo Alzedo, dando conciertos y dejando un buen nombre como compositor de música de cámara. Su prestigio probablemente le sirvió para una oferta que le hizo el gobierno boliviano y que lo motivó para que en 1833 se trasladase a Sucre, Bolivia, junto con su familia, donde ejerció el cargo de maestro del Colegio de Educandas y maestro de capilla 
de la catedral de Sucre hasta su muerte en $1856^{\circ}$. La gran mayoría de sus obras conocidas se conservan en dicha ciudad y en colecciones privadas y comprenden tanto música profana -de cámara y sinfónica-, como música sacra. El Archivo y Biblioteca Nacionales de Sucre (Bolivia) conserva en la actualidad varias composiciones suyas para diversas combinaciones de cámara. Una de ellas es el Divertimento concertante para guitarra, dos flautas $y$ cuarteto de cuerdas, op. 43 que empleamos como fuente en este estudio ${ }^{10}$.

El Divertimento consta de cinco movimientos:
1. Adagio-Allegro
2. Allegro Moderato
3. Tiempo de Vals
4. Adagio Molto
5. Allegretto

Carecemos de la fecha de composición exacta de este divertimento y es difícil determinar si lo llevó con sus pertenencias cuando salió de Arequipa o si fue compuesto en Sucre durante la estadía del maestro allí. De acuerdo con el análisis del inventario de obras que el mismo compositor realizó en los últimos años de su vida y comparándolo con informaciones existentes en la prensa musical limeña de la década de 1830, José Manuel Izquierdo especula que las obras de cámara y las sinfonías contenidas entre los Op. 47 y 57 podrían haber sido creadas antes de 1830, lo que le daría al Op. 43 una datación correspondiente con la época en que Pedro Ximénez vivió en Arequipa ${ }^{11}$, por tanto, se trataría de una obra escrita en su época peruana. Sí es posible decir es que empleó música popular oída o inspirada en su ciudad natal para escribirlo, ya que el quinto movimiento, si bien solo titula "Alegretto", tiene elementos melódicos y rítmicos claramente relacionados con géneros orales de la música tradicional arequipeña de la primera mitad del siglo XIX.

En el presente artículo, se hará un breve estudio del tercer y del quinto movimiento, el "Tiempo de vals" y el "Allegretto", que son los que contienen referencias a géneros tradicionales surperuanos del primer tercio del siglo XIX y se relacionarán sus características con las de dichos géneros.

\footnotetext{
${ }^{9}$ Para mayor información sobre sus datos biográficos, José Manuel Izquierdo König y Zoila Vega Salvatierra: "Nuevos aportes acerca de la vida del compositor peruano-boliviano Pedro Ximénez Abrill Tirado (1784-1856)", Revista Musical Chilena, 71, 227, 2017, pp. 48-78.

${ }^{10}$ Conservada en el catálogo del archivo con el número ABNB 1305. La autora desea agradecer al Archivo y Biblioteca Nacionales de Bolivia, sede Sucre, que se le haya facilitado el material para la redacción del presente artículo.

${ }^{11}$ J. M. Izquierdo Köning: Being a Composer in the Andes during the Age of Revolutions. Choices and Appropriations in the Music of José Bernardo Alzedo and Pedro Ximénez Abrill Tirado, tesis doctoral, University of Cambridge, 2017, p. 117-118 (https://doi.org/10.17863/CAM.12398).
} 


\section{El tercer movimiento del Divertimento concertante, op. 43: "Tiempo de vals"}

\section{Antecedentes sobre el minué y el vals en Sudamérica}

El tercer movimiento del Divertimento concertante, op. 43 lleva el título de "Tiempo deVals" aunque posee algunas características de la conocida danza de tres tiempos, se trata más de un minueto que de un vals en regla. Ambas danzas, en un momento determinado, compitieron en popularidad en los salones sudamericanos. El minué, de acuerdo con Carlos Vega, ya se encuentra en Sudamérica en 1735 y para 1840, era la danza más importante de los salones puesto que servía de apertura a las fiestas y saraos ${ }^{12}$. Para Remi Hess, el vals nace como expresión de ciertos sectores alemanes que se oponen (consciente o inconscientemente) a la cultura francesa aristocrática representada por el minué ${ }^{13}$. El rápido ascenso del vals se ve beneficiado por una serie de eventos histórico sociales en la Europa que lo ve nacer, como la Revolución Francesa, el fin del Antiguo Régimen, el ascenso al poder de la burguesía y el advenimiento de la pareja en la sociedad europea como forma de danza, pero también como modo de vida y de entidad social ${ }^{14}$.

En Sudamérica, el vals tiene una aparición temprana. Está citado en Montevideo y Buenos Aires desde $1804^{15}$ y Carlos Vega asegura que alrededor de 1810 y 1815 ya se encuentra en toda América del Sur ${ }^{16}$. Al Perú llega casi por la misma época, pues está tempranamente documentado en Arequipa por Antonio Pereyra y Ruiz en su Noticia de la muy noble y leal ciudad de Arequipa terminada en 1816. En su folio 7 dice:"la disposición para la música y el baile es buena pero no progresan en esto por falta de maestros. Sin embargo, el minué, el wals [sic], el Bolero, el Zapateo, el Rin, la Contradanza y otros bayles de Europa los baylan bien, pero nunca dan a su cuerpo la elegancia que en los bayles propios del país"17.

Treinta años después, un visitante francés, Eugene de Sartiges, visitó Arequipa en 1833 (el mismo año en que Ximénez partió para Sucre) y por ello su testimonio es doblemente valioso para nuestro estudio:

Me acordaré siempre de un baile dado en gran parte en honor mío. [...] Una vez que llegaron los invitados, la dueña de casa fue a buscar a una señora a la que llevó al piano. [Después de una contradanza] comenzó el vals y quise valsar a la

${ }^{12}$ Carlos Vega: El origen de las danzas folklóricas, Buenos Aires, Ricordi, 1956, p. 142.

${ }^{13}$ Remi Hess: El vals. Un romanticismo revolucionario, Buenos Aires, Paidós, 2004, pp. 20-23.

${ }^{14}$ Ibid., p. 17.

${ }^{15}$ Ibid., p. 128.

${ }^{16}$ C. Vega: El origen de las danzas..., p. 81.

${ }^{17}$ Hemos empleado tanto la edición clásica de Enrique Carrión Ordóñez: Estudio de un texto de la ilustración, Lima, Pontificia Universidad Católica del Perú, 1983, p. 374, como la última edición y estudio crítico y biográfico de Manuel Hernández González: Antonio Pereyra, Noticia de Arequipa, Tenerife, Ediciones Idea, 2009, p. 39. 
alemana, como se baila en todas partes en Europa. Mi compañera, después de tres o cuatro saltos fuera de compás, declaró sin aliento que jamás había oído hablar de un movimiento de vals tan violento y que le era completamente imposible seguirme. A propósito de esto me hicieron muchas preguntas sobre el vals en Europa y me rogaron valsar como en París. Una señora más valerosa que las demás se decidió a servirme de pareja y empezamos. No habíamos recorrido la mitad del salón cuando mi compañera se detuvo de improviso y se sentó en un sillón riendo a carcajadas. Los espectadores hicieron coro y yo con ellos de buena gana. Su vals es muy lento, con muchos contoneos y está enriquecido con toda clase de movimientos de los brazos y los hombros ${ }^{18}$.

No solo el vals era más lento que el europeo. Una forma de danza considerada ya extinta en Europa se bailaba todavía en los salones arequipeños, como dice Sartiges: "El minuet está a la moda y se admiraron que yo no lo supiera bailar. Me vi obligado a jurar que jamás lo había visto a no ser en la Ópera o en algún ballet de fantasía"19.

Ya en una fecha tan tardía como 1833, Arequipa atesoraba el uso del minué. La conservación de un baile tan antiguo que en Europa era considerado caduco puede deberse a la influencia tardía del rococó clásico de la mano de Haydn, que era un músico muy apreciado en España a comienzos del siglo XIX y, por consiguiente, en sus antiguas colonias, que vivían en esa época los remanentes de un clasicismo que servía, en palabras de José Manuel Izquierdo, como un símbolo de modernidad, cosmopolitismo e ilustración ${ }^{20}$. También puede deberse la supervivencia del minué a la difusión tardía de las ideas de la Revolución Francesa en Sudamérica, ideas que acabaron con la preeminencia del minué en la Francia Revolucionaria, pero que no consiguieron lo mismo en la España imperial y sus colonias.

En la época inmediatamente posterior a la Independencia, uno de los géneros más característicos en el salón es el vals, pero es muy probable que el gusto por el minué acerque esta danza del siglo XVIII al recién importado vals europeo produciendo formas mixtas regionales, a diferencia del Viejo Mundo donde cada género encarnaba filosofias y estamentos sociales contrapuestos. El ejemplo enunciado por Pedro Ximénez en el tercer movimiento del Divertimento concertante, op. 43 muestra esta ambigüedad estilística y parece ser un ejemplo de ese vals temprano, con aires de minueto, de ritmo lento que se bailaba en los salones sudamericanos en el primer tercio del siglo XIX. En otras obras para guitarra, las únicas que se conservan en ese periodo, Ximénez cultiva indistintamente el vals y el minué con características muy semejantes entre sí.

\footnotetext{
${ }^{18}$ Eugene de Sartiges: "Arequipa”, Imagen y leyenda de Arequipa, Edgardo Rivera Martínez (comp.). Lima, Fundación Manuel Bustamante de la Fuete, 1996, pp. 273-274. El énfasis es de la autora.

${ }^{19}$ Ibid., p. 275.

20 J. M. Izquierdo Köning: Being a Composer..., p. 124.
} 
Observaciones a la obra

El “Tiempo de vals" está dividido en diecinueve secciones de ocho compases cada una, en lo que obedece la norma de la música danzada para el desplazamiento de las parejas. Las quince primeras secciones se repiten y, a partir de la decimosexta sección, en el compás 113, se inicia una reexposición que desemboca en una larga coda final.

A continuación, mostramos algunas frases en melodía y acompañamiento de este movimiento. El primer ejemplo es la melodía de la primera frase en el primer violín y muestra características muy semejantes al minueto clásico.

Violín I
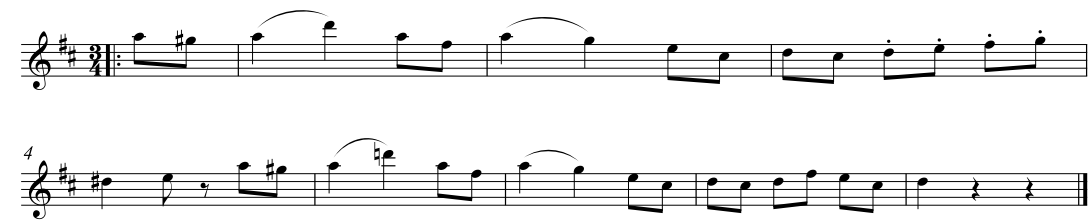

Ejemplo 1. Tercer movimiento "Tiempo de vals". Melodía de la primera frase en el violín 1, cc. 1-8

Como puede observarse, el motivo de la frase de dos corcheas y dos negras es más propio del minueto que del vals. Los finales femeninos de sub-frases y frases se corresponden con el estilo de minueto del siglo XVIII.

Los acompañamientos, por otro lado, no son típicos del vals, sino del minueto. En la primera frase, la guitarra acompaña con primer y segundo tiempo en negras.

P1
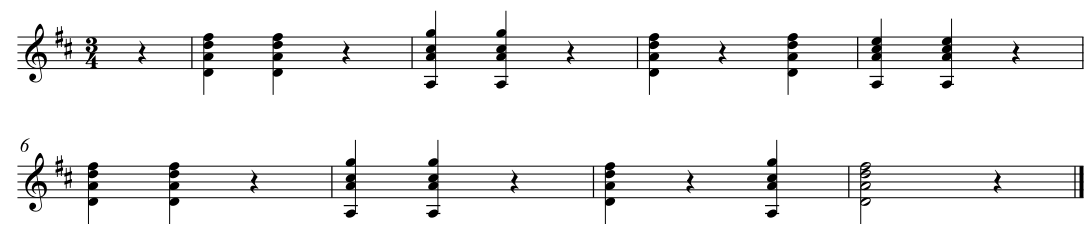

Ejemplo 2. Tercer Movimiento "Tiempo de vals". Acompañamiento de la guitarra en la primera frase, cc. 1-8

Las siguientes frases mantienen esta característica, excepto en la frase N. ${ }^{\circ} 5$ y N. ${ }^{\circ} 6$. La guitarra entra como instrumento solista con una melodía que tiene reminiscencias del minué:

P1
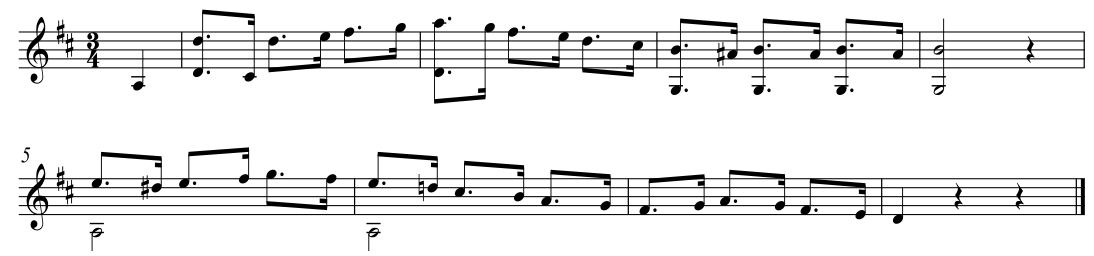

Ejemplo 3. Melodía de la guitarra en la frase 5, cc. 33-40 
A continuación, el acompañamiento del tutti, más propio del vals: primer tiempo en silencio, segundo y tercer tiempos en negras.

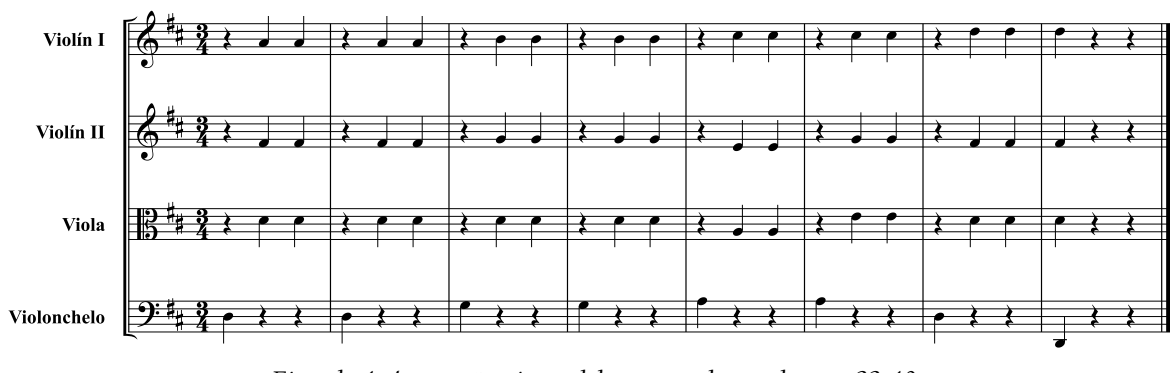

Ejemplo 4. Acompañamiento del cuarteto de cuerdas, cc. 33-40

Como carecemos de indicación metronómica exacta, la ejecución podría asumir un valor intermedio entre vals y minué.Ya hemos visto cómo el vals temprano en América era mucho más lento que su símil europeo y conservó el pulso del minué y otras características de acentuación y figuración, como lo demuestra esta obra.

Con el tiempo, este vals que se encuentra en los salones sufre modificaciones importantes hasta convertirse en un género tradicional que en la actualidad se cultiva con largueza. Según Carpio Muñoz, el vals en Arequipa siguió dos caminos: uno fue el vals de salón, con algunos ejemplos indigenistas, abordado por compositores académicos como Manuel Aguirre $(1863 ; 1951)$ o Luis Duncker $(1874 ; 1922)$, puramente instrumental y originalmente escrito para piano, bajo la forma de vals vienés que concatena varias melodías relacionadas entre sí por la tonalidad. Otro fue el vals popular, con influencia del yaraví, con letra para ser interpretado en espacios públicos y que, a semejanza del vals criollo limeño, se difundió ampliamente en sectores donde la transmisión oral cedió paso a la difusión de la música grabada, todo ello en un periodo comprendido entre finales del siglo XIX y la primera mitad del siglo XX, dando origen a lo que hoy se conoce como vals arequipeño ${ }^{21}$. De esta manera, este género fue transformándose hasta alcanzar características muy distintas del que tuvo en la primera mitad del siglo XIX.

${ }^{21}$ Juan Guillermo Carpio Muñoz: El pendón musical de Arequipa, Lima, Adrus D \& L, 2014, pp. $312-314$ 


\section{El quinto movimiento del Divertimento concertante, op. 43: "Allegretto"}

El quinto movimiento del Divertimento concertante op. 43 tiene tres secciones claramente definidas. Una primera sección en allegro escrita en tonalidad de Re menor y en compás de $6 / 8$, una segunda sección contrastante en adagio, que mantiene la tonalidad anterior y está escrita en compás de 4/4 y una tercera sección que reexpone la primera en su mismo ritmo y carácter y luego cambia a una sección en Re mayor, manteniendo el mismo compás. En la primera y tercera sección, Pedro Ximénez emplea una forma bailable de la época que podía recibir varios nombres como bailecito, cielito o gallinacito y, en la segunda, emplea un yaraví. Comenzaremos por la primera y la tercera y su relación con danzas similares de la época.

\section{La primera y la tercera sección del quinto movimiento "Allegretto"}

Se presenta como un aire danzable en modo menor, en compás de 6/8. Es muy posible que se trate de una danza de la época, bien establecida en el altiplano peruano y boliviano, con ramificaciones hasta Arequipa y su zona costera.

El movimiento comienza con un tema en menor expuesto por la guitarra y luego desarrollado por el resto de los instrumentos en cinco frases de ocho compases cada una, lo que ayuda a establecer la rigurosa métrica de la danza.
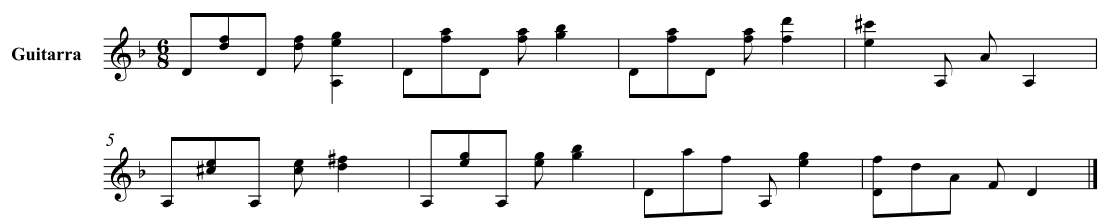

Ejemplo 5. Primer tema en la guitarra, quinto movimiento del Divertimento, op. 43, cc. 1-8

Aquí vemos la segunda frase con el tema principal encargado a las flautas
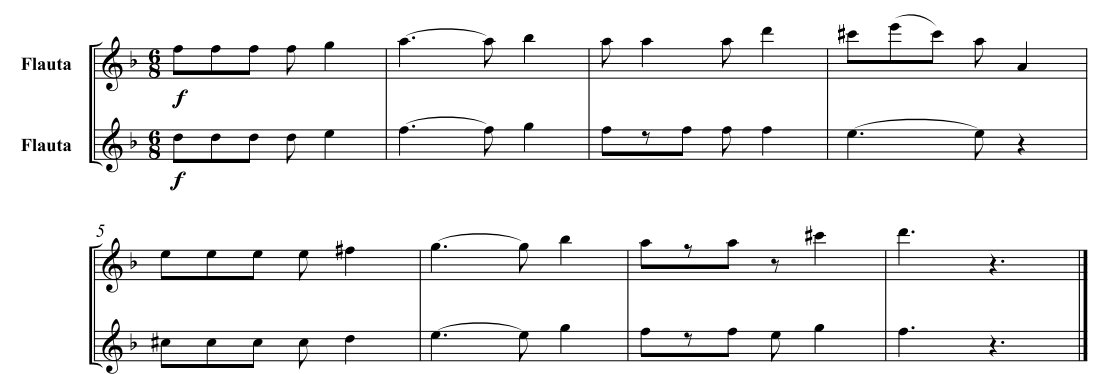

Ejemplo 6. Tema de la frase 2 en las flautas con el mismo motivo rítmico, cc. 9-16 
Después se desarrolla la segunda sección que analizaremos más adelante. En la tercera sección, se presenta una breve reexposición del primer tema. A continuación (compases 116-312), hay un cambio a modo mayor y presentación de un tema mucho más largo y desarrollado en cinco frases melódicas que sugieren un contraste o desarrollo del tema menor, pues conservan el mismo patrón rítmico hasta crear un clímax que da fin a la obra, que podría equivaler perfectamente a lo que en bailes tradicionales se conoce con el nombre de "remate".

En el siguiente ejemplo vemos cómo el motivo rítmico es muy semejante, aunque haya pasado a modo mayor.

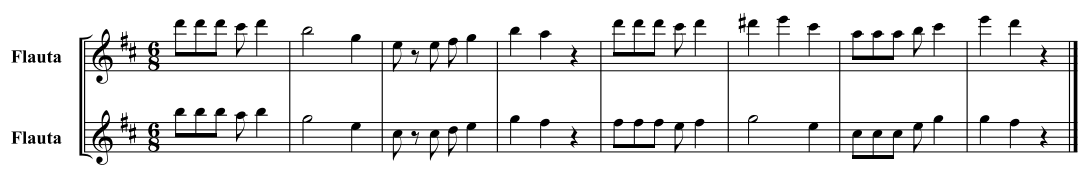

Ejemplo 7. Tema en modo mayor de la sección tercera del quinto movimiento, cc. 118-125

Como se observará, el motivo principal de cuatro corcheas y una negra es la constante de este tema, lo que lo emparenta con una danza documentada en otras fuentes a principios del siglo XIX.

\section{El baile del gallinacito (1816) y otras danzas}

En la ya mencionada Noticia de Arequipa, escrita entre 1814 y 1816, Antonio Pereyra consigna algunos ejemplos musicales de bailes y tonadas populares que se escuchaban durante la época que él vivió en esta ciudad, lo que la convierte en el primer documento histórico en consignar música arequipeña popular que hoy está perdida. Tres son los ejemplos que encontramos: Un gallinasito (sic), bayle de Arequipa en el folio 53, un Bayle del Moro en el folio 54 y un Cielito, bayle de Potosí, en el folio 55 como para demostrar la estrecha relación en cuanto a música que existía entre Arequipa y el altiplano boliviano. La Noticia no indica letra para la primera pieza, que es la que nos interesa. Está escrita en un registro muy agudo que sugiere su instrumentación para violines o flautas y al parecer se trata de una danza influenciada por el fandango andaluz, con compás doble de 3/4 y $6 / 8$, pariente de otros géneros similares muy extendidos en el resto de América Latina hasta el tiempo presente, pero que en Perú se extinguió a mediados del siglo XIX, desplazado por la zamacueca, la actual marinera y el tondero. Ahora bien, según lo registra Enrique Carrión, el gallinazo es una danza que se bailaba en Perú a comienzos del siglo XIX ${ }^{22}$. Aquí colocamos la transcripción.

${ }^{22}$ E. Carrión: Estudio de un texto..., p. 247. 
P1
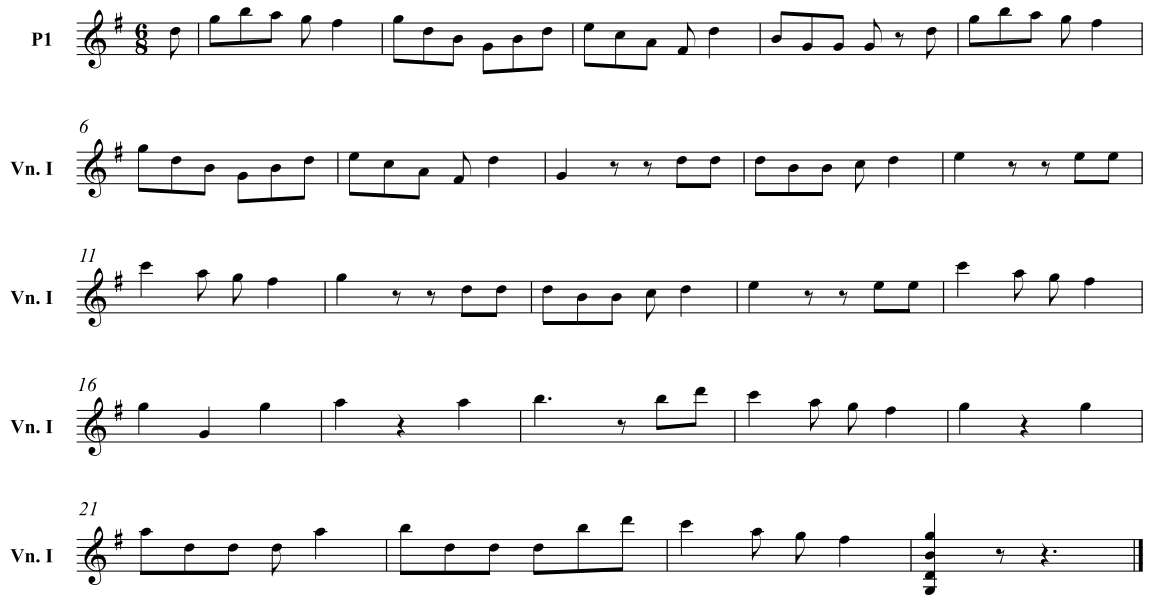

Ejemplo 8, El "baile del gallinasito" (sic) recopilado por Antonio Pereyra y Ruiz

Hay una notable semejanza de este fragmento con el del tema del $\mathrm{Di}$ vertimento. El motivo de cuatro corcheas y el uso de la hemiolia son las características comunes más llamativas.

En 1870, la casa editora Edoardo Sonzogno de Milán publicó el Álbum Sudamericano de Claudio Reblagliati, compositor genovés afincado en el Perú, y que reunía en sus páginas veintidós aires o danzas de Sudamérica que Rebagliati recogió durante sus viajes por este continente. Es uno de los primeros intentos serios por recopilar música popular en el Perú, si bien el autor no da información precisa de dónde se había recogido cada ejemplo y las circunstancias de su ejecución. Además, Rebagliati "arregló" los aires tratando de "sugetarlos (sic) a las reglas del arte" 23 , por lo que en algunos casos no cabe esperar transcripciones fidedignas o exactas. Con todo, es un documento valioso para documentar el folclore sudamericano y sobre todo peruano del siglo XIX. La última melodía consignada llamada El amor en cuarto, lleva el subtítulo de "baile arequipeño", y tiene características muy similares a las del Gallinacito arriba mencionado, especialmente en sus primeros compases de introducción y en la primera frase que va del compás 5 al 10, escrito en 6/8. A continuación se cita el tema principal:

${ }^{23}$ Claudio Rebagliati: Álbum Sudamericano: colección de bailes y cantos populares para piano solo, Fort Worth, Filarmonika, 2010, p. vi. 

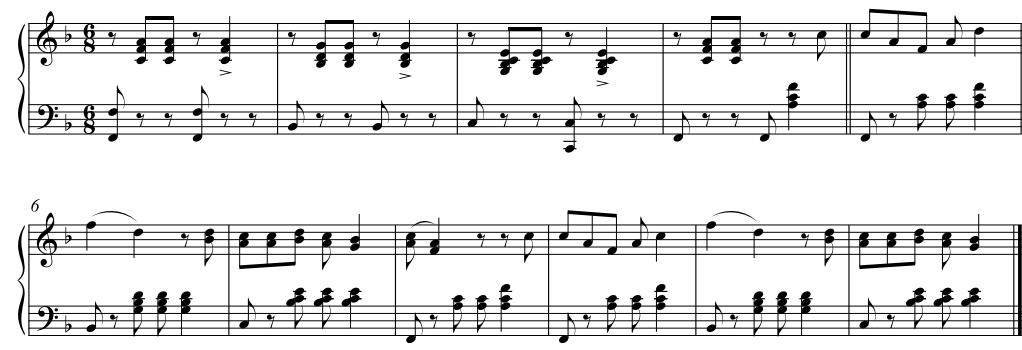

Ejemplo 9. Introducción y tema principal de El amor en cuarto recopilado por Claudio Rebagliati ${ }^{24}$

Como se observará, la característica de estos ejemplos es el uso del mismo patrón rítmico de cuatro corcheas y una negra también presente tanto en el Gallinasito, como en el tema del quinto movimiento del Divertimento.

La presencia de un motivo rítmico para los tres ejemplos es lo que nos hace suponer que tanto el Baile del gallinasito consignado por Pereyra y Ruiz, El amor en cuarto, recopilado por Claudio Rebagliati y el tema inicial del quinto movimiento del Divertimento op. 43, pertenecen al mismo género bailable, en boga en Arequipa probablemente desde finales del siglo XVIII hasta mediados del siguiente.

Otra característica de estos aires o temas, es el uso de la hemiolia, propia de ritmos ternarios con doble acentuación: en 3/4 y 6/8, acentuación binaria y ternaria propia de géneros de origen español que se diversificaron en América como el huapango mexicano, el bailecito boliviano, la cueca chilena y la chacarera argentina.Veamos cómo se presenta la hemiola en estos ejemplos:

En la segunda frase de El gallinasito vemos claros patrones de compás en hemiolia en los primeros compases, señalados con flechas.

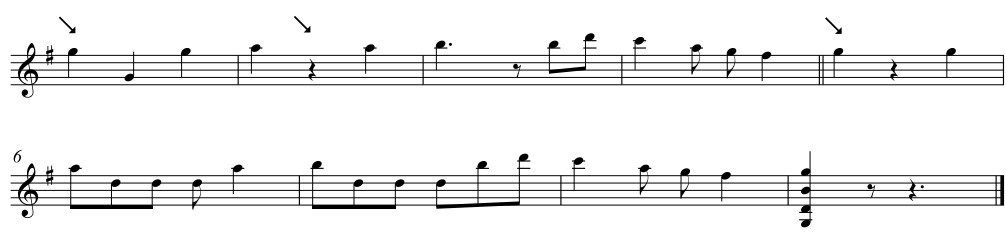

Ejemplo 10. Motivo hemiolado en la melodía de El gallinasito

\footnotetext{
${ }^{24}$ Citado con permiso de Filarmonika Music Publishing.
} 
En El amor en cuarto, tenemos la misma característica.

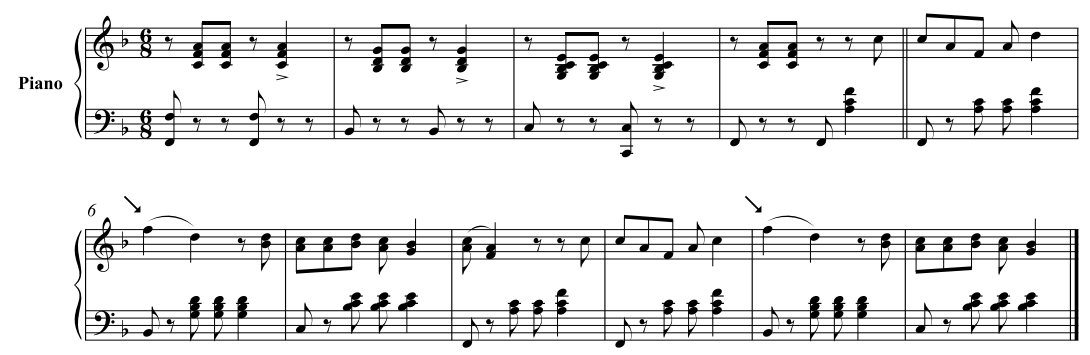

Ejemplo 11. Hemiolas en el motivo principal de El amor en cuarto

Mientras que en el Divertimento es aún más claro especialmente en el acompañamiento. En la primera frase, se expresa en la voz del violonchelo:

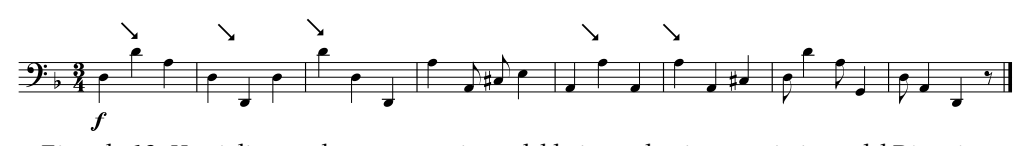

Ejemplo 12. Hemiolias en el acompañamiento del bajo en el quinto movimiento del Divertimento

En cambio, en otras el acompañamiento de las cuerdas toma la figuración en hemiolia:

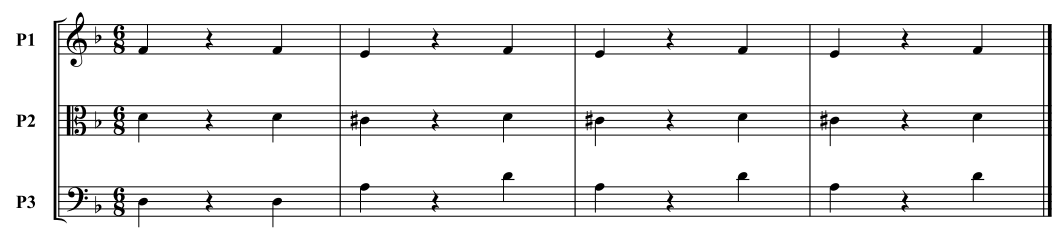

Ejemplo 13. Acompañamiento de las cuerdas medias y graves

Las melodías en mayor también presentan esa peculiaridad:

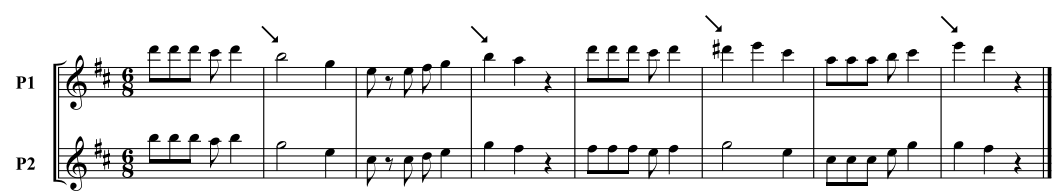

Ejemplo 14. Melodía en modo mayor en las flautas con motivos en 3/4 sobre compás de 6/8

Los patrones rítmicos expresados en estos tres ejemplos difieren notoriamente de los de la zamacueca. Si tomamos como ejemplo las zamacuecas que consigna Rebagliati en el mismo Álbum sudamericano, encontraremos una combinación de patrones rítmicos y hemiolias distintas y más complejas que las utilizadas en los fragmentos estudiados, así como síncopas y combinaciones de motivos rítmicos diferentes. Ximénez recoge un tipo 
de baile cuyo nombre desconocemos. En algún punto se le llamó gallinacito, pero también pudo recibir el nombre de bailecito de la tierra o baile de pañuelo. En qué momento desapareció es imposible de determinar. Progresivamente, fue reemplazado por la zamacueca y la marinera y, en el último tercio del siglo XIX, ya no se escuchaba. No se encuentran ejemplos vigentes en Arequipa, pero sobreviven danzas semejantes en otros lugares del continente, particularmente en Sucre y Potosí.

\section{La segunda sección: el tema contrastante (compases 48-87)}

Esta sección posee las características musicales del yaraví arequipeño, salvo por el hecho de estar escrito en 4/4. Posiblemente, el compositor lo hizo así para adaptar a un esquema más rígido el ritmo libre del yaraví debido a las licencias expresivas propias de este género. Además, la conversión a compás de 4/4 permite la construcción de frases equilibradas y simétricas (antecedentes y consecuentes) propias de la música del clasicismo. Los acompañamientos del bajo, las escalas mestizas (escalas pentáfonas anhemitónicas del quinto modo con notas de paso que ejercen funciones de escala menor) y el desarrollo de la melodía en terceras paralelas, son propias de esta canción y se incorporan sin alterarse, al punto que la transformación rítmica no hace desaparecer el carácter del género. Las frases pierden simetría, se hacen más pequeñas e irregulares, como corresponde a un género recitado o cantado que sigue la prosodia de un texto.Además, cada frase melódica entonada por las flautas, la guitarra y los violines son respondidas por la viola y el bajo en movimiento descendente en arpegio, imitando un motivo guitarrístico que es común en este canto y que recibe el nombre de bordoneo.

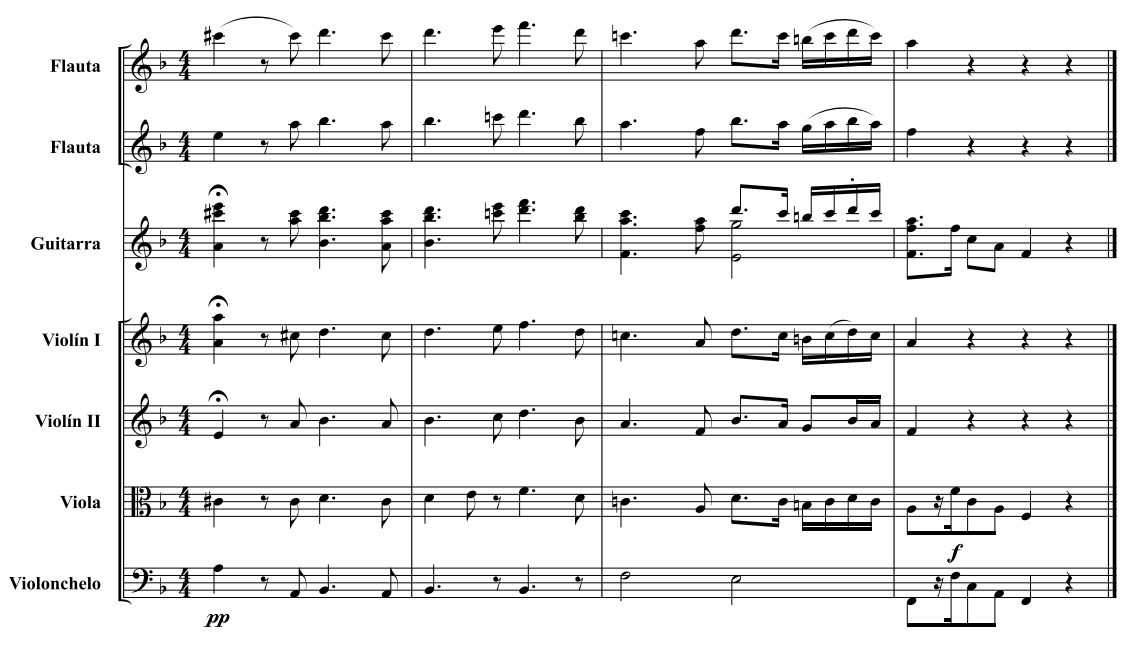

Ejemplo 15. Tema de yaraví en la sección media del quinto movimiento, cc. 48-51 


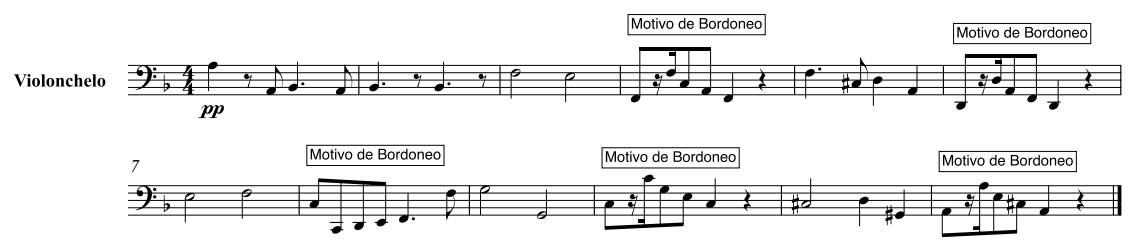

Ejemplo 16. Uso del bordoneo en el violonchelo, cc. 48-59

Conserva siempre una estructura de arpegio, para afianzar la zona armónica en la que se encuentra (tónica, dominante) y no deja de presentarla al final de cada frase, que, en este caso, no es de estructura regular, pues cada frase puede contar con tres, cuatro o más compases.

La melodía, en cambio, se halla en las flautas, que la llevan por terceras, imitando las voces que cantan. Pueden detectarse falsas relaciones cromáticas cuando la voz superior emplea, dentro de una escala menor armónica, el sexto y el séptimo grado alterados al ascender y sin alteraciones al descender, mientras que la segunda voz emplea la escala menor sin alteraciones, salvo para la sensible cuando hace cadencia sobre la tónica. Esto puede verse en el siguiente ejemplo, en el que las flechas señalan las falsas relaciones cromáticas entre las voces.
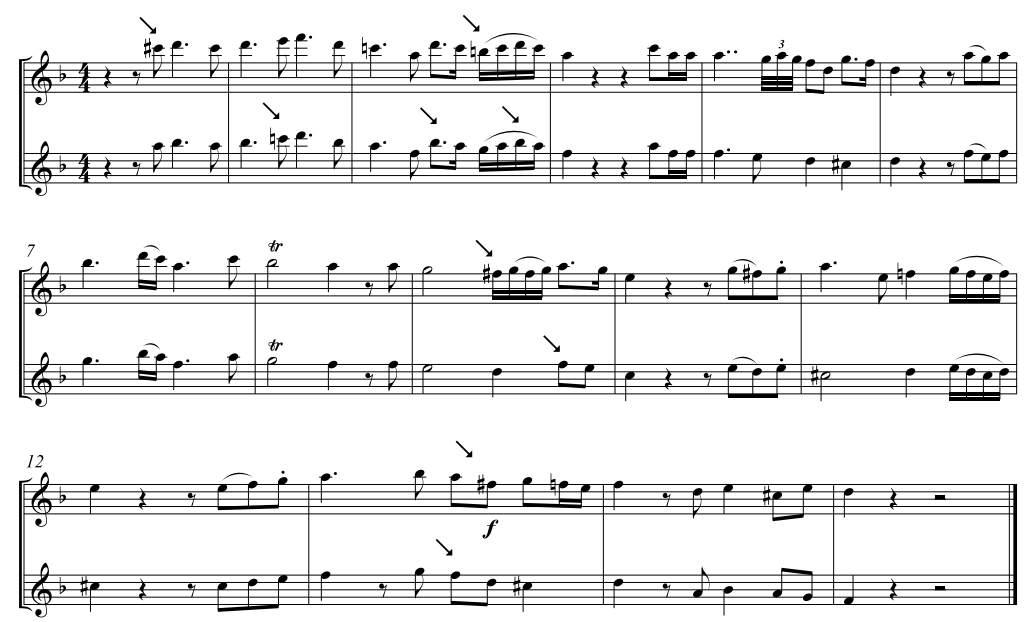

Ejemplo 17. Melodía de yaraví en las flautas, cc. 48-62

El yaraví del siglo XIX y el yaraví hoy

El yaraví es un género vocal tradicional peruano que tiene equivalentes con otras formas musicales en otras regiones andinas, tanto en Bolivia, como en Argentina y Ecuador. Está tan diversificado que se conocen 
variantes regionales claramente diferenciadas como la ayacuchana, la cusqueña y la arequipeña. En la costa peruana recibe el nombre de triste y suele ir acompañado de una sección bailable, mientras que en la sierra se entona sin baile. En el caso de la ciudad de Arequipa se ha convertido en un género fuertemente asociado a la identidad local que se considera como parte del patrimonio cultural inmaterial, pese a que su uso ha ido en franco declive durante el último siglo.

Antonio Pereyra, en su ya citada Noticia, explicaba que los yaravíes eran unas canciones que los habitantes del Perú

componían llenas de dolor en que solo expresaban lamentaciones, quejas y penas. [...] Con el tiempo y la civilización pasaron estas al estrado en los que, concertando un poco más estos tristes ecos, aunque sin perder la languidez de su carácter el amante manifiesta el sentimiento de que se siente agitado, se lamenta de su suerte, y expresa a su amoroso objeto todo lo que quiere hacerle saber ${ }^{25}$.

Pereira describe cómo un género de origen oral o popular entró a los salones donde se interpretó de manera más refinada. Más adelante añade: "la guitarra se toca generalmente en los estrados y entre la plebe" 26.

Tomando en cuenta que el sacerdote escribía lo que vio entre los años de 1810 a 1815 , nos encontramos con una perfecta correspondencia entre su época y la de Ximénez, en la cual la guitarra solista no era una rareza y menos en música de cámara y sabemos con certeza que el yaraví ya se interpretaba como música de salón.

El yaraví tiene una lejana correspondencia con el harawi precolombino, el cual se diversificó y asumió diferentes usos y significados como ha estudiado Julio Mendívil27. Uno de estos usos es el yaraví mestizo que se halla mencionado en fuentes literarias del siglo XVIII, donde casi siempre se le menciona como música triste propia de indígenas, y en otros casos aparecen empleados en diversos contextos (como en el teatro y la iglesia). A finales del siglo XVIII y comienzos del XIX, las élites criollas empezaron a unir poesía amorosa con la música indígena a la que consideraban naturalmente triste y produjeron el tipo de canción con temática de amor contrariado que hoy conocemos como yaravíes, asignándole un origen y sentido indígena que creaba lazos de unión entre ambos grupos sociales, en un momento en que la Independencia hacía necesario que los criollos desarrollaran símbolos de pertenencia al territorio en que habían nacido

${ }^{25}$ A. Pereyra: Noticia..., p. 68.

26 Ibid.

27 Julio Mendívil: "El Harawi histórico incaico y sus reminiscencias en los Andes Actuales", La memoria popular y sus transformaciones, Martin Lienhard (ed.), Frankfurt, Iberoamericana, 2000, pp. 173-183. 
para poder reclamar derechos de autodeterminación sobre él ${ }^{28}$. Enlistaremos las principales características musicales que posee el género basado en las pocas transcripciones que se han conservado y en los ejemplos que pueden escucharse en la ciudad en el presente.

Características musicales:

- Son canciones que combinan elementos expresivos de letra y música.

- Usan escala pentafónica menor con giros pentafónicos en melodía, frase y cadencia.

- Se interpretan a dúo por voces masculinas o femeninas que siguen líneas paralelas a distancia de terceras.

- En el acompañamiento se emplean las guitarras afinadas en temple de baulí2 ${ }^{29}$ y la bandurria.

- El modo es menor.

- Las cadencias de la melodía emplean alteración de la tercera menor.

- El ritmo del yaraví es libre (rubatos, calderones y acelerandos de acuerdo a las exigencias del texto).

- En ocasiones es bimodal, con la voz superior en mayor y la voz inferior en menor.

- Su compás es de $3 / 4$ o $3 / 8^{30}$.

Observemos a continuación el tema de yaraví que propone Ximénez en el Divertimento:

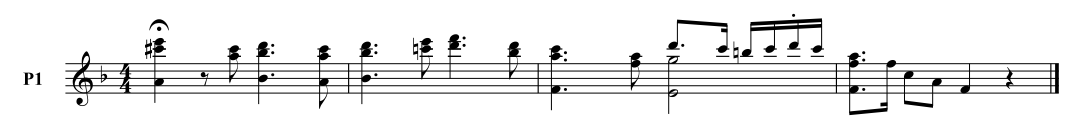

Ejemplo 18. Tema de yaraví en la sección media del quinto movimiento. Parte de guitarra

No se ha podido identificar la melodía ni compararlo con ningún yaraví arequipeño del siglo XIX o del XX que se conozca, pero la escritura ha sido alterada para poder hacerlo cuadrar dentro de un esquema formal

\footnotetext{
${ }^{28}$ Para estudios más profundos sobre el tema puede consultarse el libro de Juan Guillermo Carpio Muñoz: El yaraví arequipeño, Arequipa, La Colmena, 1976; la reedición del texto de Juan Luis Dammert: "El 'Delirio' de Melgar o el intercambio entre poesías y culturas bajo el volcán de Arequipa", Influencia y legado español en las culturas tradicionales de los Andes americanos. III Encuentro para la promoción y difusión del patrimonio folclórico de los países andinos, Bogotá, Dupligráficas, 2003, pp. 281-286; y el apartado que al tema dedica Marcela Cornejo Díaz en su estudio, Música popular tradicional del Valle del Chili, Lima, Theia, 2011.

${ }^{29}$ Baulí: Afinación de la guitarra (primera en natural, segunda en Do, tercera en Sol o Fa sostenido, cuarta en Re, quinta en Sol, sexta en Re, que imita las sonoridades del arpa indígena). Ximénez emplea esta afinación en Pasatiempo al pie del volcán, una obra escrita solo para guitarra.

${ }^{30}$ J. G. Carpio Muñoz: El pendón..., p. 100.
} 
clásico y un compás de $4 / 4$, que no es el propio del yaraví arequipeño. Emplea las terceras paralelas y las alteraciones en cada voz en las cadencias, así como el bordoneo (motivo arpegiado) en el último compás.

Para poder comprender cómo sería la escritura de la melodía de este yaraví, proponemos la misma frase en lo que sería la métrica tradicional entonada por cantores populares.
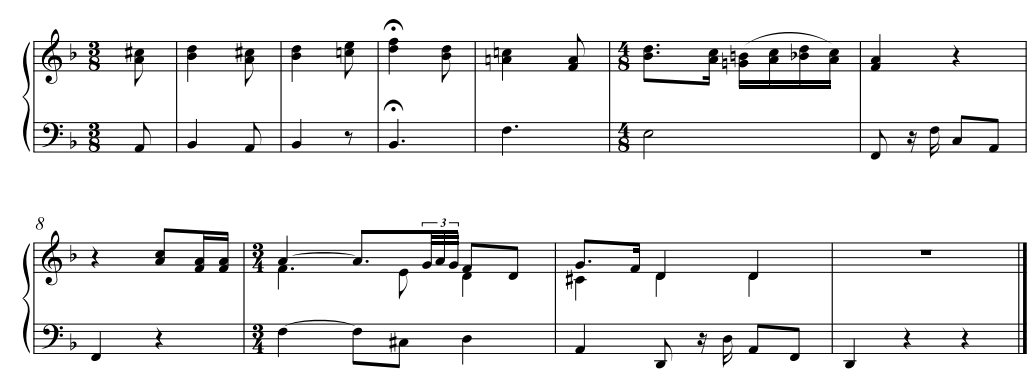

Ejemplo 19. Reconstrucción hipotética del yaraví del tema del quinto movimiento del Divertimento

Esa transformación de un compás que originalmente pudo ser de 3/4 o 3/8 parece obedecer al propósito del compositor de introducir un material contrastante no solo en carácter, sino en métrica y como el primer tema del allegretto es un compás hemioliado quizás esa sea la razón por la que el tema intermedio sea reformulado en 4/4.A pesar de la reconversión rítmica, la línea melódica mantiene varias características fundamentales, donde las flautas tienen la melodía la mayor parte del tiempo. Imitando la evolución por terceras paralelas de las voces cantadas, las flautas y a veces los violines, dibujan un perfil lleno de cromatismos que hacen una cadencia particular al final de cada frase. Las alteraciones se dan sobre todo durante la escala ascendente, característica que coincide con la señalada por Juan Guillermo Carpio Muñoz ${ }^{31}$.

Otras fuentes escritas, aunque muy posteriores, nos permiten identificar similitudes en el material empleado por Ximénez. En las transcripciones de tres yaravíes, publicadas por Tchudi y Rivero en $1851^{32}$, se usa el compás de 4/4, lo que da la idea de que quizás en algún momento el yaraví pudo tener esa métrica, pero no existen pruebas suficientes que lo avalen. Presentamos a continuación el yaraví N. ${ }^{\circ} 3$ de esta colección ${ }^{33}$ :

\footnotetext{
31 J. G. Carpio Muñoz: Arequipa, música y pueblo, Arequipa, Cordea, 1984, p. 40.

32 Mariano Eduardo De Rivero y Ustáriz, Johann Jakob Von Tschudi: Antigüedades Peruanas, Madrid, Imprenta Imperial de la Corte y del Estado, 1851, pp. 135-138.

33 Se ha omitido la introducción del original ya que se trata de una elaboración extraña a un yaraví, concebida para tocarse al piano.
} 

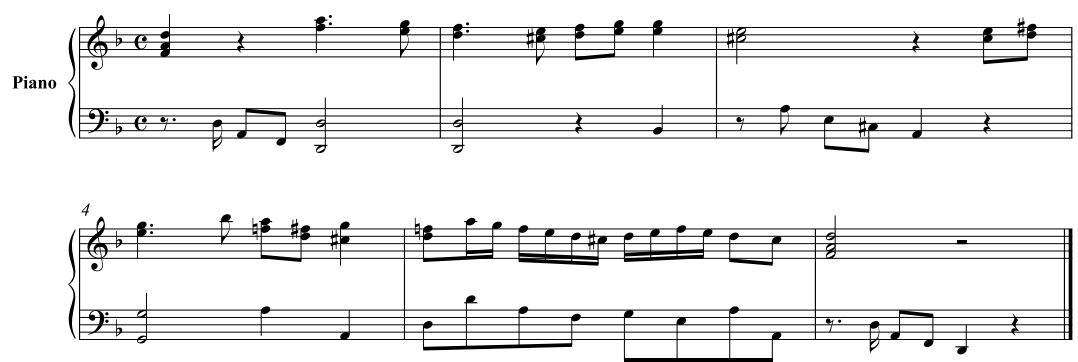

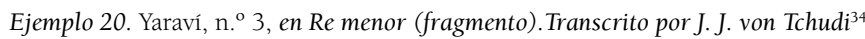

Puede observarse que la melodía va en un movimiento por terceras y que se producen las características alteraciones y las falsas relaciones de terceras entre la primera y la segunda voz, especialmente en el compás 4, entre Fa natural y Fa sostenido. En el tercer y sexto compás del fragmento, la mano izquierda tiene a su cargo el motivo del bordoneo.

Aquí mostramos un fragmento de otro yaraví recopilado por Rebagliati y publicado en 1870 en el mismo Álbum sudamericano al que aludíamos líneas arriba:

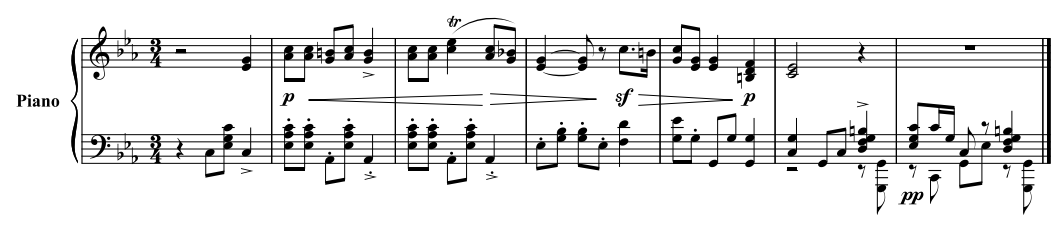

Ejemplo 21. Melodía del yaraví El pajarillo recopilado por Claudio Rebagliati

Como se observa, el compás ya está en 3/4, la melodía se sucede por terceras paralelas y aunque el acompañamiento fue modificado por Rebagliati, este yaraví es fácilmente reconocible. Todavía se canta hoy en día con el siguiente texto:

Un pajarillo cautivo se halla sin poder volar pobre de aquel pajarillo cautivo y sin libertad

El siguiente ejemplo fue recopilado y publicado en el siglo XX por el folclorista y compositor Benigno Ballón Farfán, quien fue un poco más respetuoso con las particularidades de las canciones, además de consignar la letra ${ }^{35}$.

\footnotetext{
${ }^{34}$ Johann Jakob Tchudi: Travels In Peru, During the Years 1838-1812, on the Coast, in the Sierra, across the Cordilleras and the Andes, into the Primeval Forests, Londres, Imprenta David Bogue, 1847.

35 Benigno Ballón Farfán: Cantares arequipeños, Lima, Maldonado, 1940, p. 6.
} 

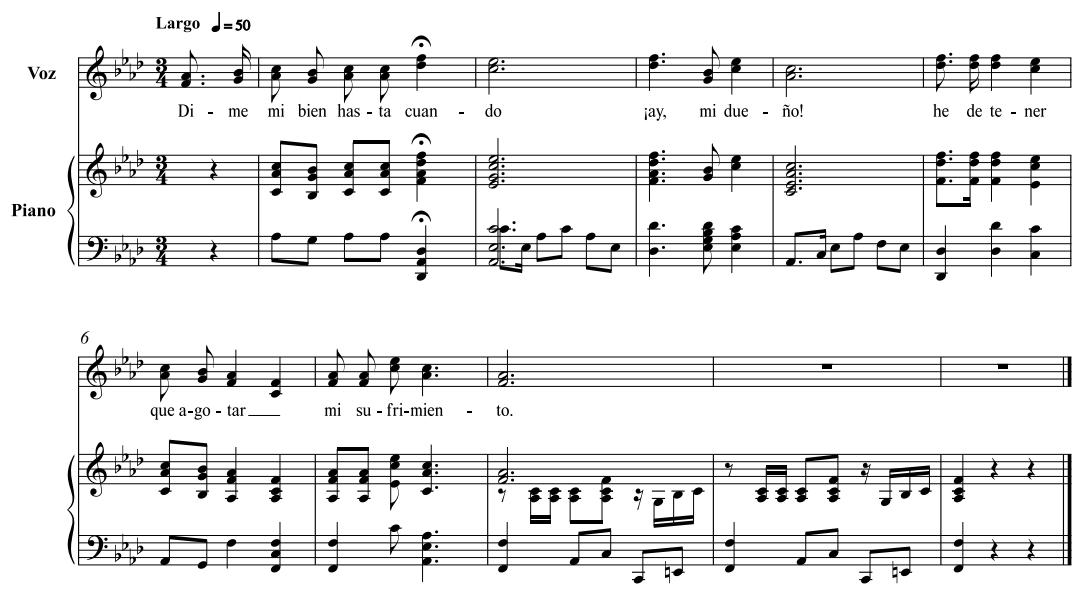

Ejemplo 22. Yaraví Delirio atribuido a Mariano Melgar. Recopilación y arreglo de Benigno Ballón Farfán

Nuevamente encontramos las terceras paralelas y el motivo de bordoneo en el bajo luego de cada verso.

\section{Algunas consideraciones}

Como podemos observar, Pedro Ximénez cita en su Divertimento, op. 43 tres géneros conocidos en su entorno en la segunda década del siglo XIX. Cabe resaltar que estas tres formas musicales, dos bailables y una cantada, tuvieron destinos muy diferentes: El vals fue sufriendo variantes y modificaciones en las décadas siguientes hasta convertirse en un género muy cultivado en la ciudad y la región. El "gallinacito", por otro lado, se extinguió durante el siglo XIX y el yaraví permaneció en el imaginario colectivo como una representación de la identidad local que expresa "lo arequipeño", cultivada por varios estamentos sociales, con características localistas y usos específicos que fueron consolidándose durante la primera mitad del siglo XIX, y transformándose continuamente merced a la transmisión oral. Asimismo, de acuerdo con Carpio $\mathrm{Muñoz}^{36}$, el yaraví es un género asociado a la Arequipa campesina del siglo XIX, en tanto el vals está muy ligado a la Arequipa urbana del siglo XX, cuando fenómenos como la migración del campo a la ciudad, el comercio internacional y la industrialización transforman profundamente la visión que la ciudad tiene de sí misma. Por esta razón, el vals arequipeño es considerado el sucesor del yaraví y se le percibe como un producto más moderno y cercano a la población actual que, sin embargo, no pierde sus nexos con el yaraví.

${ }^{36}$ J. G. Carpio Muñoz: Arequipa, música y pueblo..., p. 104. 
No es un tema menor el hecho de que los tres géneros hayan tenido distinta presencia en fuentes escritas. El Gallinacito fue consignado tempranamente en 1816 en la Noticia de Pereyra y Pacheco y el siguiente ejemplo de una danza similar es El amor en cuarto recopilada por Rebagliati en 18681870, pero aquí termina toda referencia documental. En cambio, el yaraví empieza a ser citado en 1851 en el libro Antigüedades Peruanas de Mariano Eduardo De Rivero y Ustáriz y Johann Jakob Von Tschudi y en adelante aparecerán una serie de referencias escritas que lo consignan cada vez con mayor frecuencia y a su vez, reflejan los gustos de cada época. Las primeras fuentes lo "traducen" al piano, buscando igualdad métrica, mientras que transcripciones más recientes se muestran más respetuosas con las fuentes orales que recogen. Es muy posible que la persistencia en la recopilación escrita se deba a la mayor valoración que tuvo el yaraví como vehículo expresivo del "auténtico arequipeñismo". El vals, por otro lado, es el que mayor éxito ha conocido: tanto la versión de salón, originalmente destinada al piano, como la versión cantada se han consignado en ediciones cuidadas o rústicas, recopilaciones, grabaciones y hasta concursos, convirtiéndolo en el género popular arequipeño con más acceso, bien a la edición o a la grabación, durante el siglo XX.

Peter Burke decía que en la transmisión de la memoria colectiva "los recuerdos se ven afectados por la organización social de la transmisión y por los medios empleados para la misma", pero enumera además algunos medios de transmisión de dicha memoria colectiva entre los cuales contaba las tradiciones orales y el "registro escrito", haciendo hincapié en que "dichos registros no son concreciones inocentes de recuerdos, sino más bien intentos de persuadir, de moldear la memoria de los demás" ${ }^{37}$. En este caso concreto, la partitura también es un registro escrito que carece de inocencia. No podemos saber cuál fue el grado de fidelidad con el que Pedro Ximénez usó el material tradicional para escribir este Divertimento, porque también abundan las preguntas sobre si en su tiempo el vals, el gallinacito o el yaraví contenían elementos identitarios que servían como comunicación entre creadores, intérpretes y oyentes; si eran parte de un discurso estético o incluso político y si las características musicales eran originales o adaptaciones propias del compositor. Tampoco podemos afirmar que en esta obra se citaran elementos procedentes de géneros orales debido a un propósito documental o a un deseo expreso de dejar constancia sonora de lo que se escuchaba en el entorno de aquel entonces. Las obras de Pedro Ximénez no son transcripciones con fines etnográficos, pero proporcionan información que de otra manera nos estaría vedada. De esta manera, la

${ }^{37}$ Peter Burke: Formas de historia cultural, Madrid, Alianza, 2000, p. 70. 
partitura nos da, por sí misma y por su conexión con otras fuentes, información que podemos emplear para determinar el grado de permanencia o desaparición de determinados estilos o géneros en la memoria colectiva.

Pero, además, Burke explicaba que, con respecto a la memoria histórica, esta puede abordarse como fuente histórica (la cual debe someterse a la correspondiente crítica) y como fenómeno histórico, lo que Burke llama "historia social del recuerdo". En este último caso formula algunas preguntas que nos resultan pertinentes para el caso abordado aquí: ¿Cuáles son las formas de transmisión de los recuerdos públicos y cómo han cambiado en el tiempo? ¿Cuáles son los usos de esos recuerdos, del pasado y cómo han cambiado? Y, a la inversa, ¿cuáles son los usos del olvido? ${ }^{38}$.

Si tuviéramos que aplicar estas interrogantes a nuestro tema, visto el uso que Ximénez hace de materiales de diversa procedencia y la permanencia o desaparición de los mismos en la música tradicional actual del sur peruano, la primera pregunta que se nos plantea sería ¿cómo y por qué se recuerda, se transforma o se olvida un género tradicional? E inevitablemente surgiría otra: ¿es la memoria colectiva la que selecciona qué preservar y la que descarta un género al surgir productos culturales que considere más afines? Y si es así, ¿cómo lo hace? ¿Cuáles son los mecanismos por los cuales algunos productos permanecen en el imaginario colectivo con o sin cambios y otros no? ¿Son la transmisión permanente o la conservación de productos que se consideran "finales", "únicos" y a veces hasta "verdaderos" o se trabaja (aunque sea inconscientemente) en dichos cambios? Por lo pronto estos interrogantes quedarán sin respuesta.

No solo los géneros en sí han sufrido olvido, cambio o permanencia. Resulta llamativo que, en representaciones actuales de esta obra, el público regional es capaz de reconocer e incluso sentirse identificado con el tema del yaraví, pero no le sucede lo mismo con la sección del tema bailable, ni con las formas de escritura emparentadas con el neoclasicismo (instrumentos de cuerda y aliento, frases simétricas, diálogo entre voces, estilo concertante, etc.) que sí fueron familiares para los contemporáneos de Ximénez.

Como puede observarse, son más las preguntas que las respuestas que plantea el caso presentado en este artículo, preguntas que apenas estamos empezando a formular y desde luego a responder, pero queda la idea central de que la partitura puede ser una herramienta que no solo proporcione datos técnicos, sino que ayude a construir nuevas teorizaciones sobre formas de conservación u olvido de géneros tradicionales y su potencial historia.

Recibido: 10-12-2018 Aceptado: 5-3-2018

${ }^{38}$ Ibid., pp. 68-69. 